\title{
Does Cognitive Impairment Explain Behavioral and Social Problems of Children with Neurofibromatosis Type 1?
}

\author{
Stephan C. J. Huijbregts • Leo M. J. de Sonneville
}

Received: 8 July 2010/ Accepted: 7 December 2010/Published online: 24 December 2010

(C) The Author(s) 2010. This article is published with open access at Springerlink.com

\begin{abstract}
Thirty NF1-patients (mean age 11.7 years, $\mathrm{SD}=3.3$ ) and 30 healthy controls (mean age 12.5 years, $\mathrm{SD}=3.1$ ) were assessed on social skills, autistic traits, hyperactivity-inattention, emotional problems, conduct problems, and peer problems. Cognitive control, information processing speed, and social information processing were measured using 5 computer tasks. GLM analyses of variance showed significant group differences, to the disadvantage of NF1-patients, on all measures of behavior, social functioning and cognition. General cognitive ability (a composite score of processing speed, social information processing, and cognitive control) accounted for group differences in emotional problems, whereas social information processing accounted for group differences in conduct problems. Although reductions were observed for group differences in other aspects of behavior and social functioning after control for (specific) cognitive abilities, group differences remained evident. Training of cognitive abilities may help reducing certain social and behavioral problems of children with NF1, but further refinement regarding associations between specific aspects of cognition and specific social and behavioral outcomes is required.
\end{abstract}

Keywords Neurofibromatosis Type 1 - Social functioning - Behavior problems - Information processing speed $\cdot$ Cognitive control $\cdot$ Social information processing

Edited by Petrus de Vries and Pierre Roubertoux.

S. C. J. Huijbregts $(\bowtie) \cdot$ L. M. J. de Sonneville Department of Education and Child Studies, Clinical Child and Adolescent Studies, Leiden Institute for Brain and Cognition, Leiden University, 9555, 2300 RB Leiden, The Netherlands e-mail: shuijbregts@fsw.leidenuniv.nl

\section{Introduction}

Neurofibromatosis Type 1 (NF1) is an autosomal dominant genetic disorder with an incidence of approximately 1 in 3000 (Moore and Denckla 2000; National Institutes of Health 1988). Clinical manifestations of NF1 include café-au-lait spots, intertriginous freckling, Lisch nodules (i.e. pigmented iris hamartomas or freckling in the iris), neurofibromas (i.e. Schwann cell tumors), optic pathway gliomas, and distinctive bone lesions (e.g., short stature or dystrophic scoliosis) (Williams et al. 2009). The NFI-gene is located on chromosome 17 (17q11.2). Neurofibromin, the protein product of this gene, is involved in Ras GTPase activation (Costa and Silva 2002). Ras GTPase downregulates Ras, a family of proteins involved in cell proliferation and differentiation. Thus, lack of Neurofibromin due to $N F 1$-gene defects may lead to a lack of inhibitory control over Ras. Increased formation, migration, and differentiation of neurons may result in structural and functional brain abnormalities associated with cognitive impairment (Costa and Silva 2002; Hyman et al. 2007; Moore et al. 2000; Steen et al. 2001). Cognitive impairment, the most common complication in NF1, has been observed in many cognitive domains, but becomes more apparent when more cognitive control is required (Huijbregts et al. 2010a; Rowbotham et al. 2009). Cognitive deficits are strongly associated with academic underachievement (Coudé et al. 2007), which, in turn, has been suggested to underlie social difficulties in NF1 (Coudé et al. 2006). The primary question of the present study is whether cognitive deficits underlie social impairments in NF1.

The brain abnormalities (e.g. reduced white matter integrity, macrocephaly, abnormal gamma-aminobutyric acid (GABA(A)-activity: Cutting et al. 2002; Costa and Silva 2002; Van Engelen et al. 2008; Zamboni et al. 2007) that have been hypothesized to underlie cognitive deficits 
in NF1 may also manifest themselves in social and behavioral difficulties. Adaptive functioning in complex social situations requires good communication between many different brain regions (e.g. Ochsner 2007), as do many cognitive (control) operations (e.g. Schlösser and Wagner 2006). NF1-patients experience many social and behavioral problems. These problems are of a diverse nature and include difficulties in interacting with peers, poorer social skills, and both internalizing and externalizing behavior problems (Barton and North 2004; Graf et al. 2006; Noll et al. 2007; Prinzie et al. 2003). Studies investigating relations between cognition on the one hand and social and behavioral functioning in NF1 on the other are very scarce. Descheemaeker et al. (2005) studied 17 NF1 children using socio-behavioral and cognitive measures but found no significant relations between them. Barton and North (2004) showed that the presence of ADHD was an important risk factor for poor social skills and outcomes. Coudé et al. $(2006,2007)$ noted that academic problems associated with attention deficits or ADHD may predispose children with NF1 to social difficulties. Prinzie et al. (2003) found no significant correlations between IQ and sociobehavioral factors such as conscientiousness, emotional stability, irritability, and openness to new experiences.

Thus, the evidence for cognitive deficits underlying social and behavioral problems in NF1 is mixed. One possible explanation for the fact that some studies did not find associations is the choice of cognitive constructs examined in this context. Evidence from neuroimaging and neuropsychological studies suggests that information processing speed, cognitive control, and social information processing should be considered in studies investigating associations between social and behavioral functioning and cognition in NF1 (e.g. Cutting et al. 2002; Huijbregts et al. 2010a, b; Hyman et al. 2007; Rowbotham et al. 2009; Steen et al. 2001). Another issue is that certain sociobehavioral outcomes appear to have received insufficient attention. Like Tuberous Sclerosis, fragile X, and disorders associated with phosphatase and tensin homo (PTEN) mutations, NF1 signals through the mTOR signaling pathway, and it has been suggested that, together, these disorders represent between 10 and $20 \%$ of autism spectrum disorders (ASD) (De Vries 2010). ASD may represent one highly prevalent neuropsychiatric phenotype of NF1. Hyperactivity-inattention may represent another. We suggest that these neuropsychiatric phenotypes should be considered social-behavioral manifestations of NF1 (rather than intermediate phenotypes), which, in turn, could be predicted by cognitive deficits.

Thus, the aims of the present study were to find out (1) whether group differences between NF1-patients and healthy controls could be confirmed for different measures of social functioning and behavior, and (2) whether cognitive control, social information processing, information processing speed or general cognitive competence could explain possible group differences.

\section{Method}

Participants

Children and adolescents with NF1 (12 boys, 18 girls; mean age 11.7 years ( $\mathrm{SD}=3.3$, range: $6.9-17.4$ years), were recruited through the Dutch Neurofibromatosis Association. All NF1 participants fulfilled the diagnostic criteria specified by the National Institutes of Health Consensus Conference (1988). Mean age of diagnosis was 3.0 years $(\mathrm{SD}=2.3$ years $)$. Fourteen cases were confirmed to be familial; 12 patients had the sporadic variant, and for four NF1-patients the variant was unknown. Written informed consent was obtained from both parents and participants, with parents verifying willingness to participate in those under the age of 12. The control group (11 boys, 19 girls) (mean age 12.5 years $(\mathrm{SD}=3.1$, range 6.0-17.3 years) consisted predominantly of unaffected siblings of the participants with NF1 $(n=22)$, supplemented with a number of their friends $(n=8)$. Ethical approval for this study was granted by Leiden University's Education and Child Studies Ethics Committee.

\section{Measures}

Social functioning/behavior problems

In this study, parents informed on social functioning and behavior problems of NF1-patients and controls through a number of questionnaires. The Social Responsiveness Scale (SRS) (Constantino 2002) was used to assess autistic traits, the Strengths and Difficulties Questionnaire (SDQ) (Goodman 1997) to assess hyperactivity-inattention, conduct problems, emotional problems, and peer problems, and the Social Skills Rating System (SSRS) (Gresham and Elliot 1990) to assess social skills.

The SRS consists of 65 items rated on a 4-point Likert scale (from $1=$ never true to $4=$ almost always true). There are five dimensions: social awareness, social cognition, social communication (capacity for reciprocal social responses), social motivation (including social anxiety/ avoidance), and autistic mannerisms (characteristic autistic preoccupations/traits). Higher scores on these scales indicate greater severity of social impairment. The SRS has an internal consistency of between .91 and .97 (Cronbach's $\alpha$ ). 
SRS total score (i.e. the sum of scores on all 5 dimensions) was used in statistical analyses.

The SSRS measures social skills using the following dimensions: Self-Control, Assertion, Cooperation, and Responsibility. The 38 items are rated on a 3-point Likert scale according to frequency of occurrence (never, sometimes, very often) over the last 6 months. The SSRS has an internal consistency of .87-.90 (Cronbach's alpha). The SSRS total score (i.e. the sum of scores on all 4 dimensions) was used in statistical analyses.

The SDQ is a short questionnaire with 25 items and contains five dimensions: Emotional Problems, Conduct Problems, Hyperactivity/inattention, Peer Problems, and Prosocial Behavior (not used here). The items were rated on a 3-point Likert scale (not true, somewhat true, certainly true). The SDQ has satisfactory internal consistency (mean Cronbach's $\alpha$ for different dimensions $=.73$ ), and good criterion validity.

\section{Cognitive functioning}

Five tests from the Amsterdam Neuropsychological Tasks (ANT) (De Sonneville 1999) were used to assess speed of information processing, cognitive control, and social information processing. Test-retest reliability, construct-, criterion-, and discriminant validity of the computerized ANT-tasks are satisfactory and have extensively been described and illustrated elsewhere (Huijbregts et al. 2010a, b; Rowbotham et al. 2009). The tasks used for this study were described in more detail in these papers as well, but a short description of each will be provided here.

In order to measure processing speed a task called Baseline Speed was used, where participants had to respond as quickly as possible, by pressing a mouse button, when a fixation cross presented at the center of the computer screen changed into a square. Participants first performed the task with their non-dominant hand and then repeated it with their dominant hand (32 trials for each hand). Processing speed was the mean RT (in ms) of leftand right-hand responses.

Cognitive control was operationalized as performance on a working memory task and a task measuring inhibitory control. The working memory task (Memory Search 2D Objects: MS2D) requires participants to remember target figures characterized by two specific features (color and shape, e.g. a blue square). Each trial shows four figures on the screen: participants press the yes-button (i.e. a response with the index finger of the dominant hand) when a target is present in the display and the no-button (a response with the index finger of the non-preferred hand) when no target figure is present. Performance in part 2 of the task (48 trials) compared to performance in part 1 (48 trials) of the task provides a cognitive control measure as WM-load is higher in task part 2. The task measuring inhibitory control was the Response Organization Arrows (ROA). The stimulus is an arrow (left- or right-pointing) and the color of the arrow determines which type of response is required. In "compatible mapping" (part 1, green arrow, 40 trials), the left-key is pressed when the arrow points to the left, and the right-key is pressed when the arrow points to the right. For "incompatible mapping" (part 2, red arrow, 40 trials), the opposite should be done. The difference in accuracy between task part 1 and part 2 is considered a measure of cognitive (inhibitory) control.

Social information processing was measured using tasks called Identification of Facial Emotions (IFE) and Matching of Facial Emotions (MFE). IFE measures the ability to identify emotions from facial expressions. Participants had to press the yes-button when the emotion expressed on the computer screen matched a particular target emotion (i.e. happy, sad, angry or fear) and the no-button when it did not (40 trials for each condition/emotion, 20 of which represented the target emotion). MFE measured the ability to match a set emotions presented on the computer screen (again using happy, sad, angry and fearful expressions) (160 trials) (see Huijbregts et al. 2010b).

\section{Disease severity}

Parents filled out a questionnaire, containing items from NF1-severity scales introduced by Riccardi (1982), and adapted by Ablon (1999) and Noll et al. (2007). "Yes" or "no"-answers were required to questions about the presence of NF1-symptomatology (motor difficulties, scoliosis, eyesight problems, cutaneous neurofibromas, tumors (plexiforms or optic glioma), café-au-lait spots, cosmetic difficulties, psychological problems, learning difficulties, and ADHD-status (as diagnosed by a child psychiatrist, and including medication-status) and other behavior problems). The sum of the "yes"-answers of all items except "ADHDstatus", "other behavior problems", "psychological problems", and "learning difficulties" (which were considered behavioral, social and cognitive outcomes in the present study) was used as a measure for disease severity.

\section{Statistical analyses}

First, correlations between cognitive outcomes that were supposedly in the same domain (cognitive control; social information processing) were examined. In case these were significantly related, the two measures for each domain were transformed into one by calculating the mean of standardized error scores. Secondly, it was examined, in the same manner as described above, whether it was possible to form one measure to indicate general cognitive ability. 
Next, group differences in social and behavioral outcomes as well as cognitive outcomes were examined using General Linear Model (GLM) analyses of variance. In case particular cognitive outcomes discriminated between NF1patients and controls, and they were related to social and behavioral outcomes, they were introduced to the analyses of social and behavioral outcomes as covariates. The cognitive variable that had the strongest correlation with any particular social or behavioral outcome was chosen to serve as a covariate in GLM analyses of covariance. In a further step, age and index of disease severity were entered as additional covariates if related to both the selected cognitive covariate and the social or behavioral outcomes.

\section{Results}

Preliminary analyses

The two measures of cognitive control (inhibitory control and working memory) were significantly related ( $r=.37$, $p=.002)$, as were the two social information processing measures (identification of facial emotions and matching of facial emotions: $r=.79, p<.001$ ), so one cognitive control measure and one social information processing score were created using the standardized error rates on the tasks. The resulting cognitive control and social information scores were also significantly related to each other ( $r=.42, p<.001)$, as well as to information processing speed $(r=.33, p=.005$ and $r=.50, p<.001$, respectively), which allowed the creation of one (standardized) score representing general cognitive ability.

Next, associations were established between measures of cognitive ability and social and behavioral outcome measures (Table 1). In general, there were significant associations indicating more social and behavioral problems when there were more cognitive difficulties. Whereas for autistic traits, social skills and emotional problems the strongest relations were observed with the total cognition score, conduct problems and peer problems had stronger relations with social information processing. Hyperactivity-inattention was related only to cognitive control.

There was a strong improvement with age for information processing speed $(r=-.51, p<.001)$, cognitive control $(r=-.35, p=.003)$, social information processing $(r=-.45, p<.001)$, and the total cognition score $(r=-.57, p<.001)$. With respect to social functioning and behavior, however, age was only significantly related to hyperactivity-inattention $(r=-.31, p=.008)$ and conduct problems $(r=-.29, p=.012)$, indicating a reduction of such problems with increasing age. There were no significant associations between the severity index (excluding psychological, learning, ADHD-status and behavioral problems) and social and behavioral outcomes. There were significant associations between disease severity and cognition (total cognition: $r=.33, p=.044$; social information processing: $r=.32, p=.049$; processing speed: $r=.50, p=.003$ ).

Based on these results, subsequent analyses were performed with total cognition score as a covariate for autistic traits, social skills and emotional problems, with social information processing as a covariate for conduct problems and peer problems, and with cognitive control as covariate for analyses of hyperactivity-inattention. Age was only entered in the analyses of hyperactivity-inattention and conduct problems and, because a lack of significant associations with outcome measures, the severity index was not entered in further analyses.

\section{Group differences}

NF1-patients scored significantly lower than controls on cognitive control $\left[F(1,58)=12.0, p=.001, \eta_{\mathrm{p}}^{2}=.17\right]$ and social information processing $[F=8.1, p=.006$, $\left.\eta_{\mathrm{p}}{ }^{2}=.12\right]$. They were also slower than their healthy counterparts $\left[F=7.1, p=.010, \eta_{\mathrm{p}}{ }^{2}=.11\right]$ and had a lower overall cognition score $[F=15.5, \quad p<.001$, $\left.\eta_{\mathrm{p}}^{2}=.21\right]$.

Table 1 Correlations between cognition and social and behavioral measures

\begin{tabular}{lcccc}
\hline & $\begin{array}{l}\text { Information } \\
\text { processing speed }\end{array}$ & $\begin{array}{l}\text { Cognitive } \\
\text { control }\end{array}$ & $\begin{array}{l}\text { Social information } \\
\text { processing }\end{array}$ & $\begin{array}{l}\text { Total cognition } \\
\text { score }\end{array}$ \\
\hline Autistic traits & $.43^{* *}$ & $.37^{*}$ & $.52^{* *}$ & $.56^{* *}$ \\
Emotional problems & $.39^{* *}$ & $.27^{*}$ & $.36^{*}$ & $.44^{* *}$ \\
Social skills & -.21 & $-.26^{*}$ & $-.24^{*}$ & $-.30^{*}$ \\
Hyperactivity-inattention & .03 & $.23^{*}$ & .08 & .13 \\
Conduct problems & .15 & .18 & $.38^{* *}$ & $.30^{*}$ \\
Peer problems & .20 & -.01 & $.38^{* *}$ & $.26^{*}$ \\
\hline
\end{tabular}

$* p \leq .05$. ** $p \leq .001$; Cognition scores represent $\mathrm{RT}$ or error rate, so higher scores correspond to poorer performance 
Furthermore, NF1-patients differed, to their disadvantage, from controls on all behavioral and social outcome measures (see Table 2).

Total cognition explained a significant proportion of the variance in autistic traits $\left[F=9.3, p=.003, \eta_{\mathrm{p}}{ }^{2}=.14\right]$. After covarying for total cognition, the group difference regarding autistic traits reduced but remained significant. Similarly, group differences regarding social skills reduced but remained significant after control for total cognition (Table 2).

For hyperactivity-inattention, covarying for cognitive control reduced the group difference, which again remained significant. Adding age, which did have a significant unique contribution to hyperactivity-inattention $\left[F(1,56)=4.3, p=.043, \eta_{\mathrm{p}}{ }^{2}=.07\right]$, did not attenuate group differences towards non-significance either. For conduct problems, statistical control for social information processing reduced the group difference to such an extent that it became non-significant. Social information processing had a unique contribution to conduct problems $\left[F=5.8, p=.020, \eta_{\mathrm{p}}{ }^{2}=.09\right]$. Adding age did not change these results, and it did not have a unique contribution itself.

For emotional problems, the difference between NF1patients and controls became non-significant after statistical control for total cognition. Total cognition itself had a significant contribution to the variance in emotional problems $\left[F=7.3, p=.009, \eta_{\mathrm{p}}{ }^{2}=.11\right]$. Finally, for peer problems, control for social information processing reduced the group difference although it remained significant. Social information processing did have a unique contribution to peer problems $[F=4.1, p=.047$, $\left.\eta_{\mathrm{p}}{ }^{2}=.07\right]$. Figure 1 illustrates that the greatest reductions in group differences after control for cognitive abilities were observed for conduct problems and emotional problems, where effect sizes (more than) halved.

\section{Discussion}

The results of this study showed that emotional problems of NF1-patients could be explained by general cognitive ability (a composite score of processing speed, social information processing, and cognitive control), and that conduct problems could be explained by social information processing deficits. These are important results as they show that improvement of (specific aspects of) cognition may help reducing behavior problems of children with NF1 to such an extent that differences with healthy controls are no longer apparent. General cognitive ability also significantly contributed to social responsiveness (or a lack of autistic traits), whereas social information processing was important for the existence of peer problems. For these socio-behavioral outcomes, however, group differences between NF1-patients and controls remained significant after statistical control for cognitive abilities.

A possible explanation for the fact that group differences remained evident for autistic traits, peer problems, social skills and hyperactivity-inattention is that aspects of cognition that might be particularly important for specific social and behavioral problems were not measured here. For example, autism is characterized not only by poor emotion recognition and EF-deficits, but also by a lack of central coherence, impaired local-global perception, and some aspects of social cognition that were not measured here, such as joint attention and Theory of Mind (Baron-Cohen 2008;

Table 2 Group differences between children with Neurofibromatosis Type 1 and controls on social and behavioral outcome measures before and after statistical control for cognitive abilities

\begin{tabular}{|c|c|c|c|c|c|c|c|c|}
\hline & \multirow{2}{*}{$\begin{array}{l}\text { NF1 }(N=30) \\
\text { Mean }(\mathrm{SD})\end{array}$} & \multirow{2}{*}{$\begin{array}{l}\text { Controls }(N=30) \\
\text { Mean }(\mathrm{SD})\end{array}$} & \multicolumn{3}{|c|}{ GLM ANOVA without covariates } & \multicolumn{3}{|c|}{ GLM ANOVA with covariates } \\
\hline & & & $F(1,58)$ & $p$ & $\eta_{\mathrm{p}}^{2}$ & $F(1,57)$ & $p$ & $\eta_{\mathrm{p}}^{2}$ \\
\hline $\mathrm{SRS}^{\mathrm{a}}$ & $59.4(23.4)$ & $21.4(9.6)$ & 67.8 & $<.001$ & .54 & 41.2 & $<.001$ & .42 \\
\hline $\mathrm{SSRS}^{\mathrm{b}}$ & $52.0(12.1)$ & $61.2(8.0)$ & 12.0 & .001 & .17 & 6.8 & .012 & .11 \\
\hline Hyperactivity-inattention $^{c}$ & $3.1(3.2)$ & $1.2(1.7)$ & 8.5 & .005 & .13 & 5.5 & .023 & .09 \\
\hline Conduct problems ${ }^{\mathrm{d}}$ & $1.2(1.6)$ & $.4(.7)$ & 6.5 & .014 & .10 & 2.7 & .106 & .05 \\
\hline Emotional problems ${ }^{\mathrm{e}}$ & $3.0(2.5)$ & $1.5(1.4)$ & 7.8 & .007 & .12 & 1.9 & .179 & .03 \\
\hline Peer problems ${ }^{\mathrm{f}}$ & $2.7(1.9)$ & $1.1(1.1)$ & 16.8 & $<.001$ & .22 & 10.4 & .002 & .15 \\
\hline
\end{tabular}

${ }^{a}$ SRS Social Responsiveness Scale, total score (social awareness + social cognition + social communication + social motivation + autistic mannerisms). Covariate: total cognition (derived from processing speed, social information processing and cognitive control)

b SSRS Social Skills Rating System, total score (self-control + assertion + cooperation + responsibility). Covariate: total cognition

c Hyperactivity-inattention score from Strengths and Difficulties Questionnaire. Covariate: cognitive control

d Conduct problems score from Strengths and Difficulties Questionnaire. Covariate: social information processing

e Emotional problems score from Strengths and Difficulties Questionnaire. Covariate: total cognition

f Peer problems score from Strengths and Difficulties Questionnaire. Covariate: social information processing 


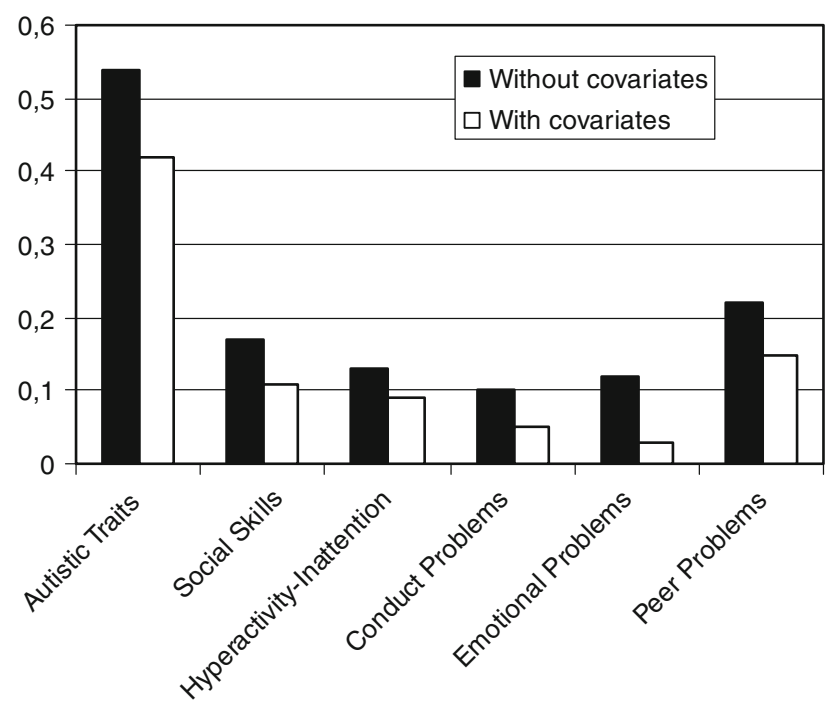

Fig. 1 Partial effect sizes of group differences (Neurofibromatosis Type 1 versus controls) before and after control for cognitive skills

Hamilton 2009; Happé and Booth 2008; Mundy et al. 2009), and for hyperactivity-inattention, reward/loss sensitivity, motor activity, sustained attention, and response variability may have more explanatory value (cf. Sergeant et al. 2003).

Despite the fact that different cognitive domains should be covered more exhaustively in relation to social and behavioral outcomes, the partial or full explanations of group differences by cognition are an important finding of this study. There may however also be social and behavioral impairments in NF1 that cannot be explained by cognitive deficits. In this (relatively small) study, measures of disease severity were not related to socio-behavioral outcomes, but severity indices that clearly distinguish features associated with, for example, cosmetic difficulties, CNS-difficulties and physiological difficulties (without neurological involvement), and that do not incorporate outcome measures (e.g. psychological, behavioral and learning problems) may help towards further explanations of social and behavioral difficulties in NF1 (cf. Barton and North 2004; Noll et al. 2007).

A second important finding is the relative magnitude of group differences for different aspects of social and behavioral functioning. Results showed that group differences were by far the largest for autistic traits (effect size $54 \%$, whereas for the other aspects of social functioning and behavior effect sizes ranged between 10 and 22\%). Although a genetic link between autism and NF1 has been suggested before (e.g. Mbarek et al. 1999) and abnormalities in the brain of NF1-patients resemble those found in autism spectrum disorders (cf. Acosta and Pearl 2004; Moore et al. 2000; Steen et al. 2001; Vaccarino et al. 2009; Williams and Minshew 2007), previous studies focused more on internalizing behavior (including emotional problems, anxiety and depression). Our findings suggest that it may be good to aim training or treatment more directly at reducing autistic traits.

Whereas some limitations of this study, such as suboptimal refinement of cognitive constructs or the NF1-severity index, are apparent from the above, there are also a number of strengths. These include an evidence-based selection of (potential) intermediate cognitive phenotypes in associations between NF1 and socio-behavioral difficulties, but also the selection of siblings as controls (see also De Vries et al. 2009). Like a number of other studies comparing sporadic and familial NF1 (e.g. Barton and North 2004), we did not find differences between the two regarding cognitive and social functioning. Sibling controls, however, also reduce the chance that group differences can be attributed to factors such as home environment, which may be affected by stress and coping with NF1, or more general factors such as socio-economic status and parenting practices. Moreover, autistic traits, which feature so prominently in the present study, and other social and behavioral outcomes are known to be highly heritable (Rutter 2000).

In summary, there are two important conclusions that can be drawn from this study. First, cognitive deficits appear to be important for the existence of social and behavioral problems in NF1. And second, autistic traits appear to be the greatest socio-behavioral problem for children and adolescents with NF1. Although more research is required to follow up both of these findings, it may still be concluded from this study that it appears worthwhile to target (specific aspects of) cognition in order to reduce (specific) social and behavioral problems of NF1-patients.

Acknowledgments The authors are grateful to Dutch Neurofibromatosis Society (NFVN), which provided an unconditional financial donation to $\mathrm{SH}$ in order to facilitate NF1-related research and helped with recruitment of study participants. A further "thank you" goes out to the NF1-patients and their families and friends for participation in this study.

Open Access This article is distributed under the terms of the Creative Commons Attribution Noncommercial License which permits any noncommercial use, distribution, and reproduction in any medium, provided the original author(s) and source are credited.

\section{References}

Ablon J (1999) Living with genetic disorder: the impact of Neurofibromatosis 1. Auburn House, Westport, CT

Acosta MT, Pearl PL (2004) Imaging data in autism: from structure to malfunction. Semin Pediatr Neurol 11:205-213

Baron-Cohen S (2008) Autism, hypersystemizing, and truth. Q J Exp Psychol (Colchester) 61:64-75

Barton B, North K (2004) Social skills of children with Neurofibromatosis Type 1. Dev Med Child Neurol 46:553-563 
Constantino JN (2002) The social responsiveness scale. Western Psychological Services, Los Angeles

Costa RM, Silva AJ (2002) Molecular and cellular mechanisms underlying the cognitive deficits associated with neurofibromatosis 1. J Child Neurol 17:622-626

Coudé FX, Mignot C, Lyonnet S, Munnich A (2006) Academic impairment is the most frequent complication of Neurofibromatosis Type-1 (NF1) in children. Behav Genet 36:660-664

Coudé FX, Mignot C, Lyonnet S, Munnich A (2007) Early grade repetition and inattention associated with Neurofibromatosis Type 1. J Atten Disord 11:101-105

Cutting LE, Cooper KL, Koth CW, Mostofsky SH, Kates WR, Denckla MB, Kaufmann WE (2002) Megalencephaly in NF1: predominantly white matter contribution and mitigation by ADHD. Neurology 59:1388-1394

De Sonneville LMJ (1999) Amsterdam Neuropsychological Tasks: a computer aided assessment programme. In: Den Brinker BPLM, Beek PJ, Brand AN, Maarse FJ, Mulder LJM (eds) Cognitive ergonomics, clinical assessment and computer assisted learning: computers in psychology. Swets and Zeitlinger, Lisse, The Netherlands, pp 187-203

De Vries PJ (2010) Targeted treatments for cognitive and neurodevelopmental disorders in Tuberous Sclerosis Complex. Neurotherapeutics 7:275-282

De Vries PJ, Gardiner J, Bolton PF (2009) Neuropsychological attention deficits in tuberous sclerosis complex (TSC). Am J Med Gen 149A:387-395

Descheemaeker MJ, Ghesquière P, Symons H, Fryns JP, Legius E (2005) Behavioural, academic and neuropsychological profile of normally gifted Neurofibromatosis type 1 children. J Intellect Disabil Res 49:33-46

Goodman R (1997) The Strengths and Difficulties Questionnaire: a research note. J Child Psychol Psychiatry 38:581-586

Graf A, Landolt MA, Mori AC, Boltshauser E (2006) Quality of life and psychological adjustment in children and adolescents with neurofibromatosis type 1. J Pediatr 149:348-353

Gresham FM, Elliot SN (1990) The social skills rating system. American Guidance Systems, Circle Pines, MN

Hamilton AF (2009) Goals, intentions and mental states: challenges for theories of autism. J Child Psychol Psychiatry 50:881-892

Happé FG, Booth RD (2008) The power of the positive: revisiting weak coherence in autism spectrum disorders. Q J Exp Psychol (Colchester) 61:50-63

Huijbregts SCJ, Swaab-Barneveld H, De Sonneville LMJ (2010a) Cognitive and motor control in Neurofibromatosis Type 1: influence of maturation and hyperactivity-inattention. Dev Neuropsychol 35:737-751

Huijbregts SCJ, Jahja R, De Sonneville LMJ, De Breij S, SwaabBarneveld H (2010b) Social information processing in children and adolescents with Neurofibromatosis Type 1. Dev Med Child Neurol 52:620-625

Hyman SL, Gill DS, Shores EA, Steinberg A, North KN (2007) T2hyperintensities in children with neurofibromatosis type 1 and their relationship to cognitive functioning. J Neurol Neurosurg Psychiatry 78:1088-1091

Mbarek O, Marouillat S, Martineau J, Barthélémy C, Müh J, Andres C (1999) Association study of the NF1 gene and autistic disorder. Am J Med Gen 88:729-732

Moore BD III, Denckla MB (2000) Neurofibromatosis. In: Yeates KO, Ris D, Taylor HG (eds) Textbook of pediatric neuropsychology: research, theory, and practice, 1st edn. The Guildford Press, New York, pp 149-170
Moore BD III, Slopis JM, Jackson EF, De Winter AE, Leeds NE (2000) Brain volume in children with Neurofibromatosis Type 1: relation to neuropsychological status. Neurology 54:914-920

Mundy P, Sullivan L, Mastergeorge AM (2009) A parallel and distributed-processing model of joint attention, social cognition and autism. Autism Res 2:2-21

National Institutes of Health (1988) Consensus development conference statement: Neurofibromatosis. Arch Neurol 45:575-578

Noll RB, Reiter-Purtill J, Moore BD, Schorry EK, Lovell AM, Vannatta K, Gerhardt CA (2007) Social, emotional, and behavioral functioning of children with NF1. Am J Med Genet A 143A:2261-2273

Ochsner KN (2007) How thinking controls feeling: a social cognitive neuroscience approach. In: Harmon-Jones E, Winkielman P (eds) Social Neuroscience: integrating biological and psychological explanations of social behavior. The Guildford Press, New York, pp 106-133

Prinzie P, Descheemaeker MJ, Vogels A, Cleymans T, Haselager GJT, Curfs LMG, Hellinckx W, Onghena P, Legius E, Van Lieshout CF, Fryns JP (2003) Personality profiles of children and adolescents with neurofibromatosis type 1 . Am J Med Genet A 118A: $1-7$

Riccardi VM (1982) The multiple forms of neurofibromatosis. Pediatr Rev 3:293-298

Rowbotham I, Pit-ten Cate IM, Sonuga-Barke EJS, Huijbregts SCJ (2009) Cognitive control in adolescents with neurofibromatosis type 1. Neuropsychology 23:50-60

Rutter M (2000) Genetic studies of autism: from the 1970s into the millennium. J Abnorm Child Psychol 28:3-14

Schlösser RG, Wagner Sauer (2006) Assessing the working memory network: studies with functional magnetic resonance imaging and structural equation modeling. Neuroscience 139:91-103

Sergeant JA, Geurts H, Huijbregts S, Scheres A, Oosterlaan J (2003) The top and bottom of ADHD: a neuropsychological perspective. Neurosci Biobehav Rev 27:583-592

Steen RG, Taylor JS, Langston JW, Glass JO, Brewer VR, Reddick WE, Mages R, Pivnick EK (2001) Prospective evaluation of the brain in asymptomatic children with neurofibromatosis type 1: relationship of macrocephaly to $\mathrm{T} 1$ relaxation changes and structural brain abnormalities. Am J Neuroradiol 22:810-817

Vaccarino FM, Grigorenko EL, Smith KM, Stevens HE (2009) Regulation of cerebral cortical size and neuron number by fibroblast growth factors: implication for autism. J Autism Dev Disord 39:511-520

Van Engelen SJPM, Krab LC, Moll HA, De Goede-Bolder A, Pluijm SM, Catsman-Berrevoets CE, Elgersma Y, Leguin MH (2008) Quantitative differentiation between healthy and disordered brain matter in patients with Neurofibromatosis Type I using Diffusion Tensor Imaging. Am J Neuroradiol 29:816-822

Williams DL, Minshew NJ (2007) Understanding autism and related disorders: what has imaging taught us? Neuroimaging Clin $\mathrm{N}$ Am 17:495-509

Williams VC, Lucas J, Babcock MA, Gutmann DH, Korf B, Maria BL (2009) Neurofibromatosis type 1 revisited. Pediatrics 123: 124-133

Zamboni SL, Loenneker T, Boltshauser E, Martin E, Il'yasov KA (2007) Detecting cerebral microstructural changes in adults with Neurofibromatosis Type. Am J Neuroradiol 28:773-776 